\title{
Conferencia Argentina de Educación Médica: agendas, aportes y temas emergentes
}

\author{
Argentinean Medical Education Annual Conference: agenda, contributions \\ and emerging issues.
}

María Eugenia Brissón y Amanda Galli

Sociedad Argentina de Investigación y Desarrollo de la Educación Médica (SAIDEM)

Propósito: En el presente trabajo se pretende identificar en las Conferencias Argentinas de Educación Médica (CAEMs) cuáles son los temas que -en relación a la formación de médicos- se han debatido, los que ocupan la agenda actual y los emergentes, así como establecer algunas relaciones con el movimiento internacional en ese campo, en los últimos veinte años. Material y método: Se trata de un estudio descriptivo del período 1985 - 2003. Se realizaron análisis de programas y resúmenes presentados (CAEMs 1999, 2000, 2001, 2002, 2003), búsquedas no sistemáticas en Internet, revisión documental y consultas a informantes claves.

Resultados: Temas en debate: admisión; evaluación y acreditación; cambios curriculares; selección, formación y evaluación del cuerpo docente y evaluación de la calidad de los egresados. Temas emergentes: asuntos de la agenda mundial son apenas incipientes en los debates de las CAEMs. Relaciones con el movimiento internacional: conformación de asociaciones sin fines de lucro para el desarrollo de la educación médica, participación en reuniones internacionales, desarrollo de proyectos de investigaciones, fortalecimiento de la formación de postgrado y legislación de aplicación en el campo de la educación médica.

Conclusiones: Existencia de contribuciones mutuas entre el pensamiento latinoamericano en educación

Correspondencia:

Brissón María Eugenia;

Dirección electrónica: saidem@saidem.org

Dirección postal : Austria 2277 PB.B

Buenos Aires (C1425 EGK) Argentina

Telefax: 00-54-011-4805-5965 médica y el global. En Argentina la expansión de la educación médica ha sido apreciable en los últimos veinte años. Las CAEM han servido como ámbito de convocatoria, discusión y difusión de las cuestiones de la agenda general y de temas particulares del país. Ha dado lugar al establecimiento de líneas de actividades y al inicio de investigaciones. En general, si bien prevalece una discusión basada en opiniones, se advierte, en el transcurso de los años, un mejoramiento de la calidad de las comunicaciones y de los trabajos presentados.

Palabras claves: educación médica, agenda en educación médica, conferencias de educación médica

Purpose: To study the themes explored at the Argentinian Medical Education Conferences (CAEMS) in the past twenty years, identifying those currently under debate and those that are likely to emerge in the new future; to explore their relationship with health care developments worldwide over the last twenty years.

Method and materials: A descriptive study performed between 1985 and 2003, analysing CAEM conferences, summits and programs, information from web sites; official, technical and institutional documents. In addition, key informants were also interviewed. Results: Current issues are: admission; evaluation and accreditation; curricular change; faculty selection, training and promotion; graduate quality. Emerging topics: key themes on the international agenda are making their mark in this country. Relations with the 
worldwide context: foundation of non-profit associations to develop medical education, participation in international conferences and summits; implementation of projects to conduct research, to strengthen or develop postgraduate capacity in medical education and to establish new legislation in higher education.

Conclusion: There is a two-way exchange of information between Latin America and the rest of the world in medical education. Medical education has evolved in Argentina in recent years. The CAEM provides a forum for discussion and dissemination of topical subjects, either general or domestic. In general, though the discussion is based more on opinion than on evidence, the quality of papers and communications has improved.

Palabras claves: medical education conferences, medical education, medical education agenda

\section{INTRODUCCIÓN}

En 1972, Juan César García ${ }^{1}$ planteaba a la educación médica como el proceso de producción de médicos, subordinado a la estructura económica dominante en las sociedades en que se lleva a cabo. Nociones desarrolladas posteriormente la han definido como:

"el proceso de enseñanza, aprendizaje y formación de los estudiantes, con una integración progresiva de conocimientos, experiencias, habilidades, actitudes, responsabilidades y valores de manera que finalmente puedan ejercer la medicina. Clásicamente se divide en enseñanza pregraduada, posgraduada y educación continua aunque se insiste cada vez más en que la educación médica es un continuum." (Wojtczak A., 2003, pp. s/28) ${ }^{2}$.

El papel educador del médico se remonta a los principios de la profesión, además de curar a los pacientes debía enseñar a los futuros profesionales de la medicina. A pesar de ello, la educación médica como disciplina es relativamente joven. La primera unidad fue establecida por George Miller, en el año 1960, en Búfalo, Nueva York. Luego se crearon departamentos similares en otras facultades de medicina de Estados Unidos y en los países anglosajones (Nolla Domenjó M., 2003) ${ }^{3}$.

En Argentina, considerando los últimos veinte años, se han producido algunos hechos particularmente significativos para el debate acerca de la formación de médicos y para el reconocimiento, por parte de los actores esenciales de las escuelas de medicina y organismos relacionados, de la educación médica como un campo de estudio específico. En este período, la colaboración de la Organización Panamericana de la Salud (OPS) facilitó la participación de los decanos de las facultades de medicina en reuniones internacionales, contribuyendo a la comprensión de la problemática de la educación médica en el contexto nacional e internacional y a la visualización de la necesidad de reformas curriculares en estrecha vinculación con las transformaciones de los servicios de salud. Estos elementos propiciaron la organización, por la Asociación de Facultades de Ciencias Médicas de la Argentina (AFACIME$\mathrm{RA}^{*}$ ), de la primera Conferencia Argentina de Educación Médica (CAEM) en 1999 y su instalación como evento anual hasta la fecha. Esta serie de conferencias nacionales que se conocen coloquialmente como las "CAEM", con sede rotativa, entre las distintas carreras miembros, están dirigidas a reunir en forma regular a profesionales de todo el país que trabajan en el ámbito de la educación en ciencias de la salud, especialmente en el área médica.

El presente trabajo tiene como propósito identificar, cuáles son los temas de la educación médica que se han debatido en Argentina en los últimos veinte años, cuáles son los que ocupan la agenda actual y cuáles son los emergentes, así como establecer algunas relaciones con el movimiento global en ese campo. Para ello se presentan antecedentes tales como eventos, estudios y proyectos que informan acerca de los principales debates nacionales e internacionales en educación médica desde el punto de vista de formación de los médicos y se analizan las CAEMs. Finalmente, se reflexiona acerca de los distintos aportes de las conferencias al desarrollo del campo de la educación médica en el ámbito de las carreras de medicina y se extraen algunas conclusiones.

\section{MATERIAL Y MÉTODO}

Se trata de un estudio descriptivo. Con el objeto de identificar los principales hitos nacionales e internacionales se realizó una revisión, limitada al período 1985-2003, de documentos oficiales, normas, reglamentos, publicaciones de encuentros internacionales de educación médica, publicaciones científicas de OPS representación Argentina e informes técnicos. Para el análisis de las CAEMs se estu-

* AFACIMERA en Julio de 2004 nuclea a 24 carreras de medicina, el 90\% de las carreras existentes (8 de las públicas y 16 privadas). 
diaron los programas y resúmenes de trabajos presentados como temas libres de las Conferencias de Educación Médica de la Argentina (1999, 2000, 2001, 2002, 2003). Esta información fue completada con una búsqueda no sistemática en Internet con las siguientes palabras claves: CAEM, Conferencia Argentina de Educación Médica, educación médica, carreras de medicina, AFACIMERA; FEPAFEM; entre las principales, y en los sitios de las carreras de medicina y de organismos nacionales tales como Comisión Nacional de Acreditación y Evaluación Universitaria (CONEAU), el Ministerio de Educación Ciencia y Tecnología (MECYT) y la Secretaría de Políticas Universitarias (SPU). En todos los casos, se incluyeron los sucesos fundamentales en los que se pudiera identificar alguna participación de educadores médicos argentinos. Además, se efectuaron consultas, en el lapso considerado, a los presidentes de la AFACIMERA, decanos y otros actores claves.

Los distintos temas presentados y discutidos más frecuentemente en las CAEMs se agruparon en cinco grandes categorías, construidas a posteriori de la revisión documental:

- Admisión

- Evaluación y acreditación de las carreras de medicina

- Cambios curriculares

- Selección, formación y evaluación del cuerpo docente

- Evaluación de la calidad de los egresados

Esta información se presenta en cuadros. Se especifican: a) en conferencias y paneles la presencia o ausencia de los temas agrupados en las categorías y b) en los posteres, la cantidad de trabajos referidos a ese tema en particular. Corresponde señalar que hay resúmenes que podrían clasificarse como misceláneas que no se consideraron en esta cuantificación.

\section{RESULTADOS}

\section{Relaciones con el movimiento global de educación médica}

Conformación de sociedades sin fines de lucro para el desarrollo del campo

El surgimiento de las CAEMs no es un hecho fortuito sino el resultado de una serie de eventos que impulsaron su realización. En el período considerado, 1985-2003, se identifica inicialmente una reunión de todos los decanos normalizadores de las carreras de medicina en 1985, en Vaquerías, provin- cia de Córdoba, en la que se decidió la constitución de una Asociación de Facultades de Ciencias Médicas. En 1986, se formalizó la AFACIMERA que es una asociación civil sin fines de lucro y con personería jurídica ${ }^{4}$. Hasta ese entonces, los contactos institucionales entre las facultades de ciencias de la salud eran esporádicos ${ }^{5}$. Por ello, los decanos de las carreras de medicina que existían en ese año (siete estatales y dos privadas), decidieron crearla con nueve propósitos, de los cuáles destacamos: a) Establecer propósitos y objetivos de educación médica para ser alcanzados por las distintas facultades y escuelas, adaptados a una realidad nacional y un perfil sanitario basado en las necesidades regionales; b) Crear comisiones para el estudio de programas de educación en ciencias de la salud y c) Efectuar el intercambio de experiencias en el terreno docente de la investigación científica y pedagógica para elevar el nivel de la misma en las distintas escuelas.

La AFACIMERA inmediatamente se integró la Federación Panamericana de Asociaciones de Facultades y Escuelas de Medicina (FEPAFEM), organismo académico internacional, privado, no gubernamental y sin fines de lucro, constituido en 1962 y dedicado al avance de la educación médica y de las ciencias biomédicas en la región de las Américas y el Caribe. A la fecha, está integrada por doce asociaciones nacionales de facultades de medicina que incluyen alrededor de cuatrocientas escuelas de medicina (388) y nueve (9) facultades individualmente afiliadas. Tiene como misión principal promover el desarrollo y perfeccionamiento de la educación médica en el continente (www.fepafem.org.ve).

En el año 2000 se constituyó la Sociedad Argentina de Investigación y Desarrollo de la Educación Médica (SAIDEM) asociación civil sin fines de lucro que convoca a organizaciones y profesionales comprometidos con la enseñanza e interesados en la investigación en el campo de las profesiones de la salud 6 . Desde entonces, esta sociedad presenta trabajos, coordina diferentes talleres y participa activamente en la organización de las distintas CAEMs.

Participación en reuniones internacionales y nacionales y en el desarrollo de proyectos de investigación y fortalecimiento de la educación médica

En el período analizado, el primer antecedente de encuentros internacionales es la Conferencia Mundial en Educación Médica de 1988, organizada por la World Federation of Medical Education (WFME) y en la que se firmó la denominada 
Declaración de Edimburgo. Esta Declaración sintetiza las recomendaciones que, sobre la formación en medicina, hicieron los educadores médicos del mundo. La región de las Américas participó y contribuyó en este debate, a través del Proyecto EMA (Educación Médica en América) que FEPAFEM desarrolló con soporte de la Fundación W.K Kellogg (FEPAFEM, 1989) ${ }^{7}$. El Proyecto EMA promovió el estudio de la situación de la educación médica en varios países de la región y propuso recomendaciones. El documento elaborado por FEPAFEM aportó a la Declaración de Edimburgo. La preparación del informe correspondiente a la Argentina y la participación de varios decanos en la Conferencia, se concretó con la cooperación de OPS. En esta cumbre se presentaron recomendaciones relacionadas con: la práctica médica y la política; la planificación de los recursos humanos para la salud y los sistemas de salud y sus implicancias en la educación médica; especialitas y generalistas, nuevas enfermedades crónicas; procedimientos de selección para el ingreso a las escuelas de medicina; desarrollo del cuerpo académico; ética de la formación en medicina; estrategias y métodos de enseñanza; la formación permanente; la formación multiprofesional; participación del estudiante en la planificación y evaluación de la educación médica; colaboración técnico financiero internacional; responsabilidad social de las universidades, entre los principales.

Durante el lapso 1988 - 1989, la OPS, en el marco de un proyecto regional y a solicitud de AFACIME$\mathrm{RA}$, facilitó la realización de un estudio de la situación de la educación médica en Argentina utilizando la metodología del Análisis Prospectivo (OPS, 1986) ${ }^{8}$. La publicación incluyó información sobre ocho facultades de medicina -de las nueve que funcionaban en el país en ese entonces (Galli A., 1989) ${ }^{9}$. En octubre de 1989 se realizó una reunión nacional en la Facultad de Medicina de la Universidad Nacional de Tucumán en la que se presentaron los resultados del Análisis Prospectivo y se debatieron los cambios curriculares necesarios.

Hacia 1991, empezó a constituirse una red latinoamericana de vínculos entre las carreras de medicina que formaban parte del "Proyecto UNI" de la Fundación WK Kelloggs, que promovía la participación de la universidad, de los servicios de salud y de organizaciones comunitarias en los procesos de formación del personal de salud. Veintitrés proyectos, en once países, adoptaron los principios de la "Una Nueva Iniciativa en la educación de los profesionales de ciencias de la salud: unión con la comunidad"
(Kissil M., et al, 1994) ${ }^{10}$. En Argentina sólo una carrera de medicina, la de la Universidad Nacional de Tucumán, participó de dicha propuesta.

La Cumbre Mundial de Educación Medica, de 1993, fue también realizada en Edimburgo (Federación Mundial de Educación Médica, $1993)^{11,12}$. La OPS y la FEPAFEM, presentaron un documento "Los cambios de la profesión médica y su influencia sobre la educación médica" que representaba la posición latinoamericana y que era producto de una amplia exploración entre las escuelas de medicina de la región ${ }^{13}$. La consulta latinoamericana recomendaba "continuar promoviendo un intenso debate que conduzca a las nuevas relaciones de la universidad, los servicios de salud y la sociedad como un todo." (OPS, 1994, pag 173) ${ }^{13}$.

El Encuentro Continental de Educación Médica de 1994, en Punta del Este, Uruguay, fue organizado por la Facultad de Medicina de la Universidad de la República, con la colaboración y patrocinio de OPS/OMS, FEPAFEM, Unión de Universidades de América Latina (UDUAL) y WFME, con el propósito de continuar el debate y la revisión de la posición latinoamericana sobre educación médica. Consistió en una serie de ponencias a cargo de expertos, de la gran cantidad de temas, mencionamos a modo de ejemplos: práctica médica y necesidades sociales; los desafíos éticos en la educación y en la práctica médica frente a las transformaciones sociales y tecnológicas; los cambios de la profesión médica y su influencia en la educación médica; experiencias evaluativas en educación médica; garantía de calidad en educación médica y la gestión de la calidad en educación médica ${ }^{13}$. En relación a la posición latinoamericana sobre educación médica se concluyó:

"Las instituciones educativas no pueden permanecer de espaldas a las situaciones cambiantes del contexto en el que se desenvuelven, están obligadas a responder a partir de un análisis de la realidad concreta y de la construcción de respuesta que pongan a prueba su capacidad, responsabilidad y compromiso con la sociedad." (Rodríguez M.I, 1994, pag 175) ${ }^{14}$.

En 1995, se realizó en Bogotá la Conferencia Regional de las Américas convocada por la Asociación Colombiana de Facultades de Medicina (ASCOFAME), auspiciada por la WFME, la OMS/OPS, la FEPAFEM y la Asociación Latinoamericana de Facultades de Medicina (ALAFEM), en la que se reformularon, en concordancia con las prioridades latinoamericanas, las recomendaciones de la Cumbre Mundial de 1993 (ASCOFAME, 1995) $)^{15}$. Como resultado de la consolidación 
de los debates se difundió la "Declaración de Santa Fe de Bogotá". Algunas de las cuestiones tratadas fueron: la interrelación y la necesidad de articulación entre la formación y los sistemas de salud y la búsqueda de la "Salud para Todos"; el crecimiento desproporcionado del número de escuelas (facultades) de medicina en Latinoamérica; los ámbitos de formación y los futuros escenarios de la práctica médica; la responsabilidad social de las facultades de medicina; el fortalecimiento del trabajo con el Estado; la colaboración por medio de la investigación, la docencia y la practica, el desarrollo de modelos de atención que respondan a los principios de equidad, calidad y relevancia social y, la promoción de las acciones de cooperación nacional e internacional para el progreso de la educación médica ${ }^{16}$.

En noviembre de 1996, la AFACIMERA y la Facultad de Medicina de la Universidad Nacional de Buenos Aires organizaron la "Cumbre Mundial de Decanos y Expertos en Salud y Educación Médica”, que consistió en conferencias de expertos y paneles integrados por decanos de las carreras de medicina, que expusieron sobre la salud frente a los cambios socio-económicos mundiales; tecnología informática para el cuidado de la salud y la educación médica; acreditación de las escuelas de medicina y de los profesionales de la salud; perfil profesional: médicos generalistas y especialistas; desafíos de la educación médica en Latinoamérica; formación de docentes e investigadores; interdisciplinariedad en educación médica; pautas de ingreso y perfil del ingresante; evaluación del egresado; flexibilización curricular; la educación médica superior en la integración nacional y regional.

La XV Conferencia Panamericana de Educación Médica fue realizada en Buenos Aires, en 1997, organizada por la AFACIMERA y la FEPAFEM. "La evolución y los cambios en la formación de los médicos de cara al tercer milenio" fue el asunto que convocó alrededor de cuatrocientos decanos y académicos de todo el mundo. El tema centralmente debatido fue la admisión y la selección para el ingreso a la carrera de medicina.

La XVI Conferencia Panamericana de Educación Médica se realizó en Buenos Aires en noviembre de 2001, convocada con el título "Autoevaluación y acreditación en tiempos de cambio". Fue organizada por FEPAFEM y AFACIMERA. Asistieron representantes de importantes instituciones líderes en educación médica como la WFME, la Asociación Europea de Educación Médica (AMEE) y autorida- des de las Asociaciones de Facultades de toda América (Educación Médica, vol. 6, 2003) ${ }^{17}$.

En octubre de 2001, en el marco de la XIV Conferencia de la Sociedad Española de Educación Médica (SEDEM), se emitió la Declaración de Granada (Educación Médica, vol 5, 2002) ${ }^{18}$. En está se promueve la adopción de los estándares internacionales para la acreditación de las carreras de medicina propuestos por la WFME (WFME, 2000) ${ }^{19}$. La Declaración fue suscripta, entre otros, por la FEPAFEM, la Asociación de Facultades de Medicina de Chile (ASOFAMECH), y SAIDEM (Educación Médica, vol. 1, 2002) ${ }^{20}$. Cabe destacar que en esta reunión SAIDEM presentó una comunicación en la que se comparaban los estándares internacionales con los argentinos utilizados en los procesos de acreditación de las carreras de medicina desde 1999 (Brissón M.E., et al, 2002) ${ }^{21}$.

Desarrollo de un espacio académico para la formación de postgrado en educación médica

Durante 1996, con apoyo de la W.K.Kelloggs, la AFACIMERA elaboró un programa de Maestría en Educación Médica en red entre las carreras miembros. Estableció convenios para su desarrollo con los Departamentos de Educación Médica (DEM) de la Facultad de Medicina de Illinois, Chicago, EEUU; con el Centro de Evaluación de Ciencias de la Salud de la Universidad de Laval y el DEM de la Facultad de Medicina de Toronto, ambos de Canadá y con el DEM de la Universidad de Dundee, Escocia. Entre las funciones del cuerpo directivo se contemplaba la organización de la Conferencia Anual de Educación Médica (Cunio R., 1997) ${ }^{22}$. La Facultad de Medicina de la Universidad Nacional de Tucumán ha sido la primera en implementar una maestría de este tipo. Otras propuestas de posgrados, en el campo de la educación médica, se encuentran en proceso de formulación.

Legislación con nuevos requisitos para el funcionamiento de las carreras de medicina

En el año 1995, se registró un hito muy importante en Argentina: la promulgación de Ley de Educación Superior que estableció la acreditación obligatoria de carreras que expiden títulos de profesiones reguladas por el Estado ("de riesgo"), en cuya nómina fue incluida la carrera de medicina hacia 1999 (Brissón M.E. et al., 1998, 2002; Ministerio de Educación, Ciencia y Tecnología, 1997, 1999) ${ }^{23,24,25,26}$. La AFACIMERA coordinó durante dos años la preparación de los documentos preliminares que aportaron los contenidos para la resolución ministerial (Fernández Lamarra N., 
2003 $)^{27}$. A partir de entonces, temas tales como autoevaluación, evaluación externa y acreditación, patrones y estándares, pares evaluadores, han pasado a ocupar un lugar importante en los debates universitarios en el país.

Durante los años 2000 y 2001, las carreras de medicina participaron en los procesos de acreditación voluntaria y obligatoria respectivamente, instalándose como actor indispensable la CONEAU. Y, es a partir del año 2000, que funcionarios, profesionales y asistentes técnicos de dicha agencia comienzan a tener participación en las CAEMs.

\section{CONFERENCIA ARGENTINA DE EDUCACIÓN MÉDICA: temas en debate y cuestiones emergentes}

La CAEM convoca en sus sesiones a expertos nacionales e internacionales, decanos, profesores, docentes y estudiantes que se reúnen para analizar, evaluar, intercambiar experiencias y proponer innovaciones en la enseñanza para el mejoramiento de la formación profesional. Se ha organizado en diferentes sedes como puede observarse en la tabla 1.
En las CAEMs la mayoría de asistentes han procedido principalmente de la Argentina, mientras que los expositores de las diferentes sesiones (además de los decanos) fueron representantes de escuelas de medicina latinoamericanas y de organismos internacionales (SEDEM, AMEE, WFME, FEPAFEM, WKKellogs, OPS). Por otra parte, en algunas CAEMs estuvieron presentes autoridades, docentes y estudiantes de carreras conexas como enfermería y nutrición.

Las conferencias ofrecen tres instancias para presentar y debatir los temas: a) conferencias: a cargo de invitados nacionales y extranjeros y paneles de decanos nacionales, b) talleres: en grupos se exponen y se discuten experiencias y/o son oportunidades para una capacitación intensiva en un asunto puntual y, c) posters: se exponen y presentan oralmente los temas. A partir de esto, se realiza una descripción resumida de la consideración de los temas según las categorías adoptadas.

\section{Admisión de estudiantes a la carrera de medicina}

En las conferencias/paneles y talleres se presentaron los fundamentos y/o necesidad de establecer un

Tabla 1. Participación según lugar de realización y año.

\begin{tabular}{|c|c|c|c|}
\hline Año & Lugar & Título & Participación \\
\hline 1999 & $\begin{array}{l}\text { San Miguel } \\
\text { de Tucumán }\end{array}$ & Sin título & $\begin{array}{l}\text { Inscriptos: datos no disponibles } \\
\text { Cantidad de trabajos presentados: } 99 .\end{array}$ \\
\hline 2000 & $\begin{array}{l}\text { Ciudad } \\
\text { de Mendoza }\end{array}$ & $\begin{array}{l}\text { Evaluación } \\
\text { de la calidad } \\
\text { de los egresados }\end{array}$ & $\begin{array}{l}\text { Inscriptos: más de trescientos } \\
\text { Cantidad de trabajos presentados: } 108 . \\
\text { Se firmó una declaración denominada } \\
\text { "Declaración de Mendoza". }\end{array}$ \\
\hline 2001 & $\begin{array}{l}\text { Ciudad } \\
\text { de Córdoba }\end{array}$ & Sin título & $\begin{array}{l}\text { Inscriptos: datos no disponibles. } \\
\text { Cantidad de trabajos presentados: } 107 .\end{array}$ \\
\hline 2002 & $\begin{array}{l}\text { Ciudad } \\
\text { de Corrientes }\end{array}$ & $\begin{array}{l}\text { Evaluación de } \\
\text { las competencias } \\
\text { profesionales }\end{array}$ & $\begin{array}{l}\text { Inscriptos: más de cuatrocientos. } \\
\text { Cantidad de trabajos presentados: } 162 \text {. } \\
\text { Se entregaron por primera vez los premios CAEM } \\
\text { a los mejores trabajos. }\end{array}$ \\
\hline 2003 & La Plata & $\begin{array}{l}\text { Innovaciones para } \\
\text { la calidad de } \\
\text { la educación médica. }\end{array}$ & $\begin{array}{l}\text { Inscriptos: datos no disponibles } \\
\text { Cantidad de trabajos presentados: } 202\end{array}$ \\
\hline
\end{tabular}

Fuente: Elaboración propia sobre la base de los programas y libros de resumen de las CAEM e información en las páginas web de las facultades de medicina respectivas. 
sistema de selección y se describieron las experiencias de algunas facultades. Los posters describen experiencias en la implementación de algunas modalidades de ingreso a la carrera de medicina: ciclos introductorios, ciclo de confrontación vocacional, módulos de nivelación, cursos de ingreso, tipo de exámenes implementados, rendimiento, predictores. (Tabla 2)

\section{Tabla 2. Admisión a las carreras de medicina}

\begin{tabular}{l|lllll}
\hline $\begin{array}{l}\text { Tema } \\
\text { presentado en }\end{array}$ & Años & & & & \\
\hline & 1999 & 2000 & 2001 & 2002 & 2003 \\
\hline $\begin{array}{l}\text { Conferencia } \\
\text { /panel }\end{array}$ & si & si & no & no & no \\
\hline Taller & n/c & si & si & no & no \\
\hline Poster (cantidad) & 8 & 4 & 3 & 7 & 16 \\
\hline
\end{tabular}

Fuente: Elaboración propia sobre la base de los programas y libros de resumen de los temas libres de las CAEM Notas: $n / c$ : no corresponde. En esa CAEM la modalidad de taller no fue implementada.

\section{Evaluación y acreditación de las carreras de medicina}

Las conferencias y paneles abarcaron centralmente los procesos de acreditación, las fortalezas y debilidades y las lecciones aprendidas. Los talleres estuvieron centrados en los procesos de autoevaluación, de acreditación y de revisión de los estándares y en los estándares internacionales para la acreditación. En los posters se hace referencia a la evaluación institucional y a la importancia de la evaluación para el mejoramiento de la enseñanza y a la acreditación de los postgrados. (Tabla 3 )

Tabla 3. Evaluación y acreditación de las carreras de medicina

\begin{tabular}{l|lllll}
\hline $\begin{array}{l}\text { Tema } \\
\text { presentado en }\end{array}$ & Años & & & & \\
\hline $\begin{array}{l}\text { Conferencia } \\
\text { /panel }\end{array}$ & si & si & no & si & si \\
\hline Taller & n/c & si & si & si & si \\
\hline Poster (cantidad) & 1 & 3 & 0 & 1 & 2 \\
\hline
\end{tabular}

Fuente: Elaboración propia sobre la base de los programas y libros de resumen de los temas libres de las CAEM Notas: $n / c$ : no corresponde. En esa CAEM la modalidad de taller no fue implementada.

\section{Cambios curriculares}

En las conferencias y paneles se trataron las tendencias y la necesidad de un cambio curricular global teniendo en cuenta las demandas del sector salud, de integración docente asistencial, de incrementar la responsabilidad social, de la evaluación y acreditación de los servicios para la práctica clínica, entre otros. En los talleres se abordaron temáticas más particulares tales como: (indicar significado de siglas) ABP, PFO, enseñanza de contenidos básicos de las áreas humanísticas e instrumentales y flexibilidad curricular. En los posters se expusieron experiencias sobre cambios puntuales en una cátedra o departamento de la carrera; innovaciones (implementación de ABP y de la PFO), nuevos recursos educativos, soporte informático etc. (Tabla 4)

\section{Tabla 4. Cambios curriculares}

\begin{tabular}{l|lllll}
\hline $\begin{array}{l}\text { Tema } \\
\text { presentado en }\end{array}$ & Años & & & & \\
\hline & 1999 & 2000 & 2001 & 2002 & 2003 \\
\hline $\begin{array}{l}\text { Conferencia } \\
\text { /panel }\end{array}$ & si & no & no & no & si \\
\hline Taller & n/c & si & si & si & si \\
\hline Poster (cantidad) & 52 & 47 & 69 & 79 & 73 \\
\hline
\end{tabular}

Fuente: Elaboración propia sobre la base de los programas y libros de resumen de los temas libres de las CAEM Notas: $n$ /c: no corresponde.

En esa CAEM la modalidad de taller no fue implementada.

\section{Selección, formación y evaluación del cuerpo docente}

Tanto las conferencias como los talleres abordaron los temas: evaluación del desempeño docente,

Tabla 5. Selección, formación y evaluación del cuerpo académico

\begin{tabular}{|c|c|c|c|c|c|}
\hline \multirow{2}{*}{$\begin{array}{l}\text { Tema } \\
\text { presentado en }\end{array}$} & \multicolumn{5}{|l|}{ Años } \\
\hline & 1999 & 2000 & 2001 & 2002 & 2003 \\
\hline $\begin{array}{l}\text { Conferencia } \\
\text { /panel }\end{array}$ & no & no & si & si & no \\
\hline Taller & $n / c$ & si & si & si & si \\
\hline Poster (cantidad) & 13 & 8 & 5 & 9 & 8 \\
\hline
\end{tabular}

Fuente: Elaboración propia sobre la base de los programas y libros de resumen de los temas libres de las CAEM Notas: $n / c$ : no corresponde.

En esa CAEM la modalidad de taller no fue implementada. 
mejoramiento de la calidad del cuerpo académico, formación docente del profesorado y criterios para la selección de profesores. Los posters se centraron en la descripción de las experiencias de capacitación docente pedagógica, en el análisis del rol docente/ tutor y en la implementación de encuestas de evaluación del grado de satisfacción de los alumnos con las innovaciones. (Tabla 5)

\section{Evaluación de la calidad de los egresados}

En las conferencias y talleres se desarrollaron las metodologías y/o herramientas utilizadas para garantizar la calidad del egresado, OSCE, prueba de fin de ciclo, evaluación de las competencias y reflexiones generales sobre la problemática. Sólo dos trabajos fueron presentados en forma de posters: perfil de egresados que postulan a residencias y encuesta sobre competencias adquiridas en la carrera de medicina según autopercepción de los egresados. (Tabla 6)

Tabla 6. Evaluación de la calidad del graduado

\begin{tabular}{l|lllll}
\hline $\begin{array}{l}\text { Tema } \\
\text { presentado en }\end{array}$ & Años & & & & \\
\hline & 1999 & 2000 & 2001 & 2002 & 2003 \\
\hline $\begin{array}{l}\text { Conferencia } \\
\text { /panel }\end{array}$ & no & si & no & si & no \\
\hline Taller & n/c & no & si & si & no \\
\hline Poster (cantidad) & 0 & 0 & 0 & 0 & 2 \\
\hline
\end{tabular}

Fuente: Elaboración propia sobre la base de los programas y libros de resumen de los temas libres de las CAEM Notas: $n / c$ : no corresponde. En esa CAEM la modalidad de taller no fue implementada.

\section{Sobre la base de estos hallazgos puede} resumirse para cada categoría lo siguiente:

Admisión: existe bastante preocupación sobre cómo implementar sistemas de selección que sean válidos y equitativos. Se advierte poca investigación de seguimiento del rendimiento de los alumnos en relación con los criterios de selección aplicados.

Evaluación y acreditación de las carreras de medicina: ha tenido gran efecto movilizador. Muchos trabajos hacen referencia a que se propone un cambio "porque la acreditación lo recomendó" pero no se articula con estándares específicos. Se advierte ausencia de estudios que relacionen fortalezas y debilidades de las carreras con propuestas de planes de acción para el mejoramiento y evaluación de resultados.
Cambio curricular: aparece como una declaración de principios pero sin identificación del problema a corregir. Predominan las presentaciones descriptivas y escasa investigación sobre resultados, efectos e impactos obtenidos con las innovaciones

Selección, formación y evaluación del profesorado: se declara que el cuerpo académico es un determinante de la calidad de la enseñanza, se insiste en la necesidad de la formación docente pedagógica, pero no se informan cambios sustantivos, ni planes de acción a mediano o largo plazo.

Evaluación de la calidad de los egresados: se expresa la preocupación por la relación entre lo que se enseña y "el médico que el país necesita" pero no se comunican investigaciones al respecto. Ausencia de estudios de seguimiento de egresados.

Los temas que más presencia han tenido son aquellos relacionados con cambios puntuales en el currículum, con la implementación de la práctica final obligatoria y con el desarrollo de experiencias de enseñanza basada en problemas. Por otra parte, persiste la discusión sobre la problemática de la admisión a la carrera de medicina. Según Cusminsky M., $2001^{28}$, este tema se debate con mayor o menor intensidad desde hace cuarenta y ocho años.

A partir del año 2002, se implementó el "Premio CAEM" a los dos mejores trabajos presentados. El premio ha consistido en la entrega de diplomas.

Finalmente, vale la pena mencionar los problemas encontrados para la realización del estudio, por la dificultad de acceder a datos, a memorias institucionales y conclusiones, por su inadecuado registro y archivo.

\section{DISCUSIÓN Y CONCLUSIONES}

Como lo señala Almeida M. (2001) ${ }^{29}$, existen contribuciones mutuas entre el movimiento latinoamericano y el global en el desarrollo de la educación médica. Como se observa en los últimos veinte años, Argentina ha acompañado estos progresos y la expansión de ha sido apreciable. Ejemplos de ello lo constituyen la presencia a lo largo de los años de expertos extranjeros en las reuniones nacionales realizadas, los puentes que se han comenzado a tender entre la educación médica Argentina y la latinoamericana así como también con países europeos (en particular España) y América del Norte (Canadá).

En Argentina, en el período estudiado, las agencias de cooperación técnico financiero internacionales (tales como la OPS y la W.K.Kelloggs) a través de 
distintas iniciativas han influenciado la formación de los médicos, ya sea a través de los principios propuestos y/o del financiamiento de proyectos.

En ese lapso también se produjo el surgimiento de nuevos actores, tanto de evaluación y acreditación de carreras de medicina como de desarrollo e investigación en educación médica.

En los últimos seis años las sucesivas CAEM han servido como ámbito de convocatoria, discusión y difusión de las cuestiones de la agenda global en educación médica y de temas particulares en el país, así como ha dado lugar al establecimiento de líneas de trabajo y al inicio de investigaciones. En general, si bien prevalece una discusión mayormente basada en opiniones y reflexiones, se advierte, en el transcurso de los años, que se ha ido mejorando la calidad de las comunicaciones y de los trabajos presentados. Las CAEMs resultan experiencias motivadoras e impulsoras de cambios.

La cantidad creciente de concurrentes y trabajos presentados marca una tendencia hacia la consolidación de este evento como un espacio para el debate. Se ha incrementado la participación de las carreras de medicina en las CAEMs, tanto institucional como de sus cuerpos académicos. Se observa también una mayor y gradual presencia de los estudiantes y de otros profesionales de la salud involucrados en la formación de personal sanitario.

Algunos asuntos, que aparecen insistentemente en la agenda internacional son apenas incipientes en los debates de las CAEMs. Entre los principales se identifican: enseñanza multiprofesional; validez y confiabilidad de los exámenes; evaluación de las competencias profesionales adquiridas; selección y promoción de profesores basada en la evaluación del desempeño; responsabilidad social de las facultades de medicina; financiación de la educación médica,

En síntesis, se ha avanzado en el reconocimiento del campo de estudio específico de la educación médica. Resta mucho aún por hacer para su fortalecimiento en la Argentina, principalmente en lo que se refiere al desarrollo de la investigación y de su complemento: la difusión y publicación.

\section{BIBLIOGRAFÍA}

1. García J. C. "La educación médica en América Latina" Publicación Científica No 255 Washington: OPS / OMS, 1972

2. Wojtczak A. (2003) "Glosario de términos de educación médica”. Educación Médica 2003; 6 (suplemento 2): S/21-S/50.

3. Nolla Domenjó M. (2003) "Investigación en educación médica” Educación Médica 2003; 6 (1), pp. 1-1.
4. Resolución de Personería Jurídica de la Inspección General de Justicia IGJ N 164 de febrero de 2001.

5. Binia A. "AFACIMERA". Disponible en www.fmed2.uncu. edu.ar/boletin/boletin\%20vol_1\% 20num_18.htm

6. Resolución de Personería Jurídica de la Inspección General Justicia $\mathrm{N}^{\circ} 001385$ de personería jurídica de SAIDEM, diciembre de 2000

7. Federación Panamericana de Asociaciones de Facultades de Medicina "Educación Médica en las Américas. Proyecto. EMA" Río de Janeiro, Brasil: Oficina del Proyecto EMA, Asociación Brasilera de Educación Médica, 1989

8. Organización Panamericana de la Salud "Análisis prospectivo de la Educación Médica. Metodología de autoevaluación.” Serie de Desarrollo de Recursos Humanos No 72 Washington: OPS / OMS, 1986

9. Galli A. (1989) "Situación Actual de la Educación Médica en la República Argentina" Publicación N²4. Argentina: OPS/OMS, 1989

10. Kisil M., Chaves M. (Eds) "Programa UNI. Una nueva iniciativa en la Educación de los profesionales de la Salud." San Pablo, Brasil: Fundación W. K. K. Kellogg, 1994

11. Federación Mundial de Educación Méica "Recomendaciones" en: "Cumbre Mundial de Educación Médica en Edimburgo" Montevideo, Uruguay: Facultad de Medicina, Universidad de la República, 1993

12. "Cumbre mundial de educación médica. Declaración Edimburgo 1993”. Rev Cubana Educ Med Super 2000;14(3): 270-83

13. Organización Panamericana de la salud "Encuentro continental de educación médica." Montevideo, Uruguay: Facultad de Medicina de la Universidad de la República - OPS / OMS, 1994.

14. Rodríguez M.I. "Toma de posición frente a las experiencias evaluativos en educación médica" en : Organización Panamericana de la Salud "Encuentro continental de educación médica." Montevideo, Uruguay: Facultad de Medicina de la Universidad de la República - OPS / OMS, 1994, 153:178

15. Asociación Colombiana de Facultades de Medicina "Conferencia Mundial de Educación Médica. Memorias.” Santa Fe de Bogotá, Colombia: AS.CO.FA.ME., 1995

16. "Declaración de Santa Fe de Bogotá." Disponible en http:// jagua.cfg.sld.cu/ aldo/diplomado/web2/pages/docs/modulo1/6.\%20DeclaraciondeSantaFedeBogota.doc

17. "Conferencia Panamericana de Educación Médica 2001. Autoevaluación y acreditación en tiempos de cambio." Educación Médica 2003; 6 (suplemento 1): S/6- S/78

18. "XV Congreso de la Sociedad Española de Educación Médica. Granada, 24 - 26 de octubre de 2001" Revista Educación Médica 2002, 5 (1): 1-1

19. World Federation of Medical Education Office (2000) "WFME Task Force para la definición de estándares internacionales para la educación médica de pregrado. Informe del Grupo de Trabajo.” Educación Médica 2000; 3 (4): 158-169 
20. "La Declaración de Granada" Revista Educación Médica 2002, 5 (1): 3-5

21. Brissón M.E., Galli A., Morera M.I. "Análisis comparativo de patrones y estándares de acreditación de las carreras de medicina de la Argentina y los propuestos por la World Federation of Medical Education.” Educación Médica Educación Médica 2002; 5 (3): 143-154

22. Cunio R., Galli A., Brisson M.E. "Informe final. Proyecto de Maestría en Educación Médica de AFACIMERA" en: Proyecto UNI Tucumán Bs As., Argentina: AFACIMERA, 1997

23. Brissón M.E., Morera M.I. (2002) "Acreditación de carreras de medicina. Legitimidad, legalidad y consenso." Educación Médica 2002; 5 (1): 13-21.

24. Brissón M.E., Morera M.I. "Carreras de riesgo: el caso de Medicina. Metodología, procesos y productos en la formulación de los patrones y estándares previstos por la Ley de Educación Superior.” Argentina: Editorial La Prensa Médica, 1998.
25. Ministerio de Cultura y Educación - Argentina "Ley de Educación Superior No 24.521 y decretos reglamentarios" Buenos Aires, Argentina, Ministerio de Cultura y Educación Secretaría de Políticas Universitarias, 1997

26. Ministerio de Cultura y Educación - Argentina "Resolución 535 /99" Buenos Aires, Argentina Ministerio de Cultura y Educación, 1999

27. Fernandez Lamarra N. "Evaluación y la acreditación en la Educación Superior Argentina." Ponencia presentada en el Segundo Congreso de Administración Pública. Sociedad, Estado y Administración, 2003

28. Cusminsky M. "Ingreso en Medicina: un debate que no es nuevo." Diario El Día. La Plata 26 de julio 2001

29. Almeida J.M. "Perspective on South America: the Latin American contribution to the world movement in medical education” Medical Education, 2001, 35: 796-799 